\author{
Denis Foschini ${ }^{1}$ \\ Jonato Prestes ${ }^{2}$ \\ Richard Diego Leite ${ }^{2}$ \\ Gerson do Santos Leite Li, $^{3,4}$ \\ Felipe Fedrizzi Donatto ${ }^{3}$ \\ Christiano Bertoldo Urtado ${ }^{5,6}$ \\ Bianca Trovello Ramallo ${ }^{1}$
}

\title{
RESPOSTAS HORMONAIS, IMUNOLÓGICAS E ENZIMÁTICAS AGUDAS A UMA PARTIDA DE BASQUETEBOL
}

\author{
ACUTE HORMONAL, IMMUNOLOGICAL AND ENZYMATIC RESPONSES \\ TO A BASKETBALL GAME
}

\begin{abstract}
RESUMO
O objetivo do estudo foi analisar as respostas hormonais, imunológicas e enzimáticas agudas a uma partida de basquetebol em jogadores profissionais desta modalidade. A amostra foi composta de oito atletas de basquetebol, praticantes a pelo menos quatro anos. Foi realizada uma partida de duração total de 40 minutos com simulação real, sendo dividida por dois tempos de 20 minutos e um intervalo de 10 minutos entre os tempos. As amostras sanguíneas foram colhidas antes e imediatamente após a partida (20 ml pelo sistema de tubos a vácuo). As variáveis analisadas foram: hormônios testosterona e cortisol, leucócitos totais, neutrófilos, linfócitos, monócitos e as enzimas creatina kinase (CK) e lactato desidrogenase (LDH). Para análise estatística, foi utilizada estatística descritiva e Teste t de Student pareado com $p \leq 0,05$. A concentração pré $(13,34 \mathrm{nmol} / \mathrm{L}$ e $301,97 \mathrm{nmol} / \mathrm{L})$ e pós $(17,34 \mathrm{nmol} / \mathrm{L}$ e $395,91 \mathrm{nmol} / \mathrm{L})$ de testosterona e cortisol foram estatisticamente diferentes, com maiores valores após o jogo para ambos os hormônios respectivamente. $A$ contagem das células imunes apresentou diferença significante nos leucócitos totais $(6393,75 \mathrm{nmol} / \mathrm{L}$ e $9158,75 \mathrm{nmol} / \mathrm{L})$ e neutrófilos (3532,5 nmol/L e 6392,62 nmol/L) com maiores valores após a partida. As variáveis enzimáticas analisadas não apresentaram diferenças significativas. Portanto, dos marcadores analisados, testosterona e cortisol mostraram aumento acentuado após a partida, o mesmo comportamento foi verificado para os leucócitos totais e neutrófilos.
\end{abstract}

Palavras-chave: Basquetebol; Treinamento desportivo; Dosagens bioquímicas.

\begin{abstract}
The objective of the present study was to analyze the acute hormonal, immunological and enzymatic responses of professional basketball players to a basketball game. The sample was composed of eight basketball athletes, with a minimum of 4 years' experience in basketball. A real game was simulated with a total duration of 40 minutes, divided into two halves of 20 minutes each and an interval of 10 minutes between halves. Blood samples were collected before and immediately after the game $(20 \mathrm{ml}$, vacuum tube system). The variables analyzed were: testosterone and cortisol hormones, total leukocytes, neutrophils, lymphocytes, monocytes and the enzymes creatine kinase (CK) and lactate dehydrogenase $(\mathrm{LDH})$. Statistical analysis was with descriptive statistics and the Student's $t$ test for paired samples to $p \leq 0.05$. The pre (13.34 nmol/L and $301.97 \mathrm{nmol} / \mathrm{L})$ and post game $(17.34 \mathrm{nmol} / \mathrm{L}$ and $395.91 \mathrm{nmol} / \mathrm{L})$ levels of testosterone and cortisol were statistically different, with higher levels after the game for both hormones. The immune cell counts exhibited significant differences for total leukocytes $(6393.75 \mathrm{nmol} / \mathrm{L}$ and $9158.75 \mathrm{nmol} / \mathrm{L})$ and neutrophils $(3532.5 \mathrm{nmol} / \mathrm{L}$ and $6392.62 \mathrm{nmol} / \mathrm{L})$, with levels being higher after the game. No statistical differences were observed for the enzymatic variables. Therefore, based on the markers analyzed, testosterone and cortisol exhibited pronounced increases after the game and the same behavior was observed for total leukocytes and neutrophils.
\end{abstract}

Key words: Basketball; Physical training; Biochemists dosages.

1. Universidade Metodista de São Paulo. Faculdade de Educação Física e Fisioterapia. São Paulo, SP, Brasil.

2. Universidade Federal de São Carlos. Programa de Pós-graduação em Ciências Fisiológicas.

Laboratório de Fisiologia do Exercício. São Carlos, SP, Brasil.

3. Universidade Metodista de Piracicaba. Faculdade de Ciências da Saúde, Mestrado em Educação Física, Piracicaba, SP, Brasil.

4. Faculdades Integradas Einstein de Limeira. Curso de Educação Física. Limeira, SP, Brasil.

5. Unimódulo. Curso de Educação Física. Caraguatatuba, SP, Brasil.

6. Universidade Estadual de Campinas. Programa de Pós-graduação em Fisiologia pelo Instituto de Biociências. Campinas, SP, Brasil. 


\section{INTRODUÇÃO}

A alteração dos marcadores fisiológicobioquímicos é modulada pela carga de treinamentos e jogos ${ }^{1}$, sendo constituída pelo volume, intensidade e freqüência de solicitação das atividades ${ }^{2}$. Uma sucessão inadequada da relação estímulo-pausa ao longo do processo de treinamento pode levar o atleta a ultrapassar seu limite individual de adaptação e ocasionar perda de desempenho ou mesmo overtraining $^{3}$. Para que isso não ocorra, faz-se necessário o acompanhamento de parâmetros que mostrem as adaptações fisiológicas ao treinamento.

A análise de parâmetros hormonais, imunológicos e enzimáticos, permite a monitoração das adaptações decorrentes da aplicação de diferentes intensidades de treinamento e também a detecção de condições não favoráveis que podem levar ao sobretreinamento. A atividade desportiva influencia mudanças no sistema endócrino pela modulação do processo de anabolismo e catabolismo, exercendo papel fundamental nas adaptações fisiológicas e morfológicas do sistema corporal ${ }^{4,5}$. A análise dos hormônios testosterona e cortisol podem auxiliar no controle sistemático da sobrecarga imposta por sessões de treinamento, fornecendo informações sobre os estados anabólico/ catabólico do atleta $6,7,8$.

As variáveis imunológicas também podem ser utilizadas no acompanhamento do desempenho de atletas, visto que, também ocorrem mudanças na distribuição e função da imunidade celular e humoral 9. Segundo Nieman ${ }^{10}$, a magnitude das mudanças que ocorrem no sistema imune depois de cada sessão de treinamento reflete o estresse fisiológico imposto aos atletas, ao passo que, em intensidades muito elevadas e período de recuperação insuficiente, pode ocorrer queda da resistência imunológica (imunossupressão). Prestes et $\mathrm{al}^{11}$ comentam que altas concentrações de cortisol observadas nos períodos de treinamento intenso podem suprimir várias reações inflamatórias e imunitárias.

Outro parâmetro que pode ser considerado é o enzimático, por meio das dosagens das enzimas creatina kinase (CK) e lactato desidrogenase (LDH) podem-se obter indicativos do grau de adaptação metabólica dos músculos esqueléticos ao treinamento físico. Ambas as enzimas estão envolvidas no metabolismo muscular, e suas concentrações séricas são normalmente muito baixas. No entanto, pode ocorrer aumento significativo destas enzimas após exercícios intensos, indicando que houve dano à célula muscular devido ao extravasamento da CK e LDH para o líquido extracelular ${ }^{12,13}$.

Alêm desses parâmetros, outro aspecto importante para o completo entendimento dos mecanismos envolvidos nas alterações fisiológico-bioquímicas do organismo é a verificação da resposta aguda do organismo frente a um determinado esforço físico. Concomitante a estas respostas, o organismo atua por meio de mecanismos para manter sua homeostase, fazendo-se necessário o conhecimento de variáveis fisiológicas como, por exemplo, variáveis hormonais, imunes e enzimáticas. A verificação destes parâmetros pode auxiliar na compreensão das respostas agudas frente à realização do exercício físico.

Segundo Thompson et a ${ }^{14}$, Rowbotton \& Green ${ }^{15} \mathrm{e}$ Afonso et $a^{16}{ }^{16}, 0$ exercício físico agudo pode influenciar parâmetros fisiológicos, como concentrações de lipídios sanguíneos, lipoproteínas, colesterol, pressão arterial, metabolismo da glicose, sistema imunológico e muitas outras variáveis. Somado a isso, até o presente momento, não foram encontrados na literatura trabalhos que tenham analisado todas estas variáveis associadas frente a uma partida de basquetebol em atletas adultos.

Neste sentido, é necessário o conhecimento do comportamento das variáveis hormonais, imunológicas e enzimáticas para verificação mais completa das respostas orgânicas e adaptativas do atleta frente a um evento esportivo específico.

Tendo em vista a lacuna apresentada no conhecimento destas variáveis na modalidade em questão, o objetivo deste estudo foi analisar as respostas hormonais, imunológicas e enzimáticas agudas a uma partida de basquetebol.

\section{PROCEDIMENTOS METODOLÓGICOS}

\section{Sujeitos}

Foram selecionados 10 atletas de basquetebol do sexo masculino, para as avaliações, mas somente oito participaram integralmente da partida analisada. A estatura e massa corporal média foram respectivamente de $1,93 \pm 0,14 \mathrm{~cm}$ e $92 \pm 13,4 \mathrm{~kg}$. A idade média foi de $22,6 \pm 4,5$ anos, sendo todos praticantes da modalidade a pelo menos quatro anos. O experimento foi aprovado pelo Comitê de Ética em Pesquisa da Universidade Metodista de São Paulo - CEP - UMESP (Parecer $n^{\circ}$ 074709/05). Todos os participantes foram informados detalhadamente sobre os procedimentos utilizados e concordaram em participar de maneira voluntária do estudo, assinando o termo de consentimento informado e proteção da privacidade.

\section{Características da partida de basquetebol}

A partida teve duração total de 40 minutos, sendo dividida por dois tempos de 20 minutos e intervalo de 10 minutos entre os dois tempos. A partida foi realizada entre os jogadores do próprio time, porém com simulação de todas as características de um jogo, incluindo arbitragem e presença do técnico. As amostras foram colhidas antes e logo após a partida. Na manhã das análises os atletas receberam um café da manhã padronizado por um nutricionista, seguindo as recomendações da Associação Americana dos Nutricionistas e Colégio Americano de Medicina do Esporte (ADA, 2004), visando reduzir as variações hormonais.

\section{Coletas sangüíneas}

Antes de iniciar o jogo, os indivíduos foram 
submetidos a repouso absoluto, durante 30 minutos, em seguida, foi realizada a primeira coleta de sangue através da veia antecubital pelo sistema de tubos a vácuo, sendo coletados $20 \mathrm{ml}$ de sangue. A mesma quantidade de sangue foi coletada imediatamente após o término do jogo. Ao término das coletas, o sangue foi centrifugado por 10 minutos a 5000 x g e armazenado a $-20^{\circ} \mathrm{C}$.

Determinação da concentração de creatina quinase (CK) e lactato desidrogenase (LDH)

As amostras coletadas foram processadas no equipamento Cobas Mira S, utilizando-se da metodologia cinético-espectrofotométrica, seguindo as especificações do Kit Bio System.

\section{Concentração de testosterona total e cortisol}

As dosagens de testosterona total e cortisol foram realizadas no soro pelo método de eletroquimioluminescência, seguindo as especificações do Kit Bio System. A técnica foi desenvolvida no aparelho Elecsys 2010 (Roche Diagnóstica).

\section{Hemograma}

As amostras sangüíneas coletadas antes e logo após a partida de basquetebol, foram submetidas à automação Sysmex SF-3000 (Roche Diagnóstica), o qual utiliza a metodologia de Citometria de Fluxo, obtendo os leucócitos totais e diferenciais (monócitos, neutrófilos e linfócitos).

\section{Análise estatística}

Todos os dados foram expressos, utilizando estatística descritiva (média \pm erro padrão da média). A análise estatística foi realizada inicialmente pelo teste de Shapiro-Wilk para verificar a distribuição dos dados e pelo teste de homocedasticidade (critério de Bartlett). Todas as variáveis analisadas apresentaram distribuição normal e homocedasticidade. Para verificar a diferença entre os dois momentos, foi utilizado o Teste $\mathrm{t}$ de Student pareado, adotando um nível de significância de $p \leq 0,05$. O software utilizado em todos os testes estatísticos foi o Statistica ${ }^{\circledR} 6.1$.

\section{RESULTADOS}

A concentração de testosterona apresentou aumento estatisticamente significativo de $29,98 \%$ do momento pré para o pós ( $p=0,00007)$. Na concentração do hormônio cortisol o mesmo comportamento foi verificado, ocorrendo aumento de $30,91 \%$ do momento pré para o momento pós $(p=0,018)$ (Tabela 1 e Figura 1). Já as enzimas CK e LDH apresentaram aumento de $18,74 \%$ e $20,70 \%$, respectivamente, porém não significativo do ponto de vista estatístico, quando comparado à coleta pré com a coleta pós (Tabela 1 e Figura 1).

Os números totais de leucócitos e neutrófilos aumentaram $(43,24 \%$ e $80,96 \%$, respectivamente) quando comparado o momento pré com o pós partida $(p=0,0182$ e $p=0,01097$, respectivamente) (Tabela 2 e Figura 1). Os linfócitos apresentaram um aumento de $7,41 \%$ e monócitos de $13,58 \%$, entretanto estes aumentos não foram estatisticamente diferentes entre os momentos pré e pós partida.

Tabela 1. Resultados das variáveis testosterona, cortisol, CK e LDH pré e pós partida de basquetebol.

\begin{tabular}{cccc}
\hline (nmol/L) & Pré & Pós & $p$ \\
\hline Testosterona & $13,34 \pm 1,66$ & $17,34 \pm 1,77$ & $0,00007^{*}$ \\
Cortisol & $301,97 \pm$ & $395,31 \pm$ & $0,018^{*}$ \\
& 70,60 & 99,41 & \\
CK & $777,50 \pm$ & $923,25 \pm$ & 0,65695 \\
& 206,88 & 245,70 & \\
LDH & $506,00 \pm$ & $610,75 \pm$ & 0,11969 \\
& 37,24 & 51,06 & 0,190 \\
\hline
\end{tabular}

Valores expressos pela Média \pm Erro padrão da média. Pré $=$ coleta antes da partida, Pós= coleta imediatamente após a partida. *Diferença significativa dos valores pré em relação aos valores pós-partida $(p \leq 0,05)$.

Tabela 2. Resultados das células imunes pré e pós partida de basquetebol.

\begin{tabular}{cccc}
\hline (nmol/L) & Pré & Pós & $P$ \\
\hline Leucócitos & $6393,75 \pm$ & $9158,75 \pm$ & $0,0182^{*}$ \\
totais & 599,88 & 842,85 & \\
Neutrófilos & $3532,50 \pm$ & $6392,62 \pm$ & $0,01097^{*}$ \\
& 523,44 & 823,96 & \\
Linfócitos & $2080,62 \pm$ & $2234,87 \pm$ & 0,45032 \\
& 127,01 & 152,70 & \\
Monócitos & $663,62 \pm$ & $753,75 \pm$ & 0,35279 \\
\hline
\end{tabular}

Valores expressos pela Média \pm Erro padrão da média. Pré= coleta antes da partida, Pós= coleta imediatamente após a partida. *Diferença significativa dos valores pré em relação aos valores pós-partida $(p \leq 0,05)$.

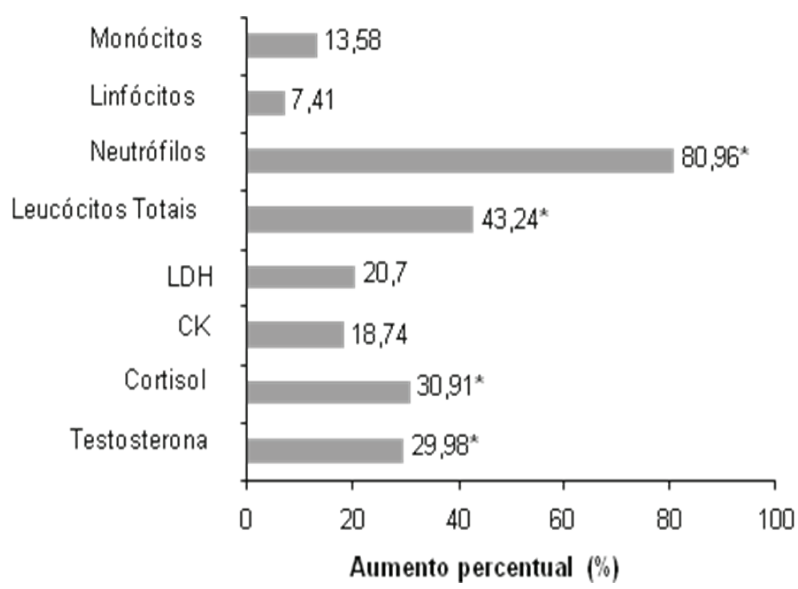

Figura 1. Aumentos percentuais nas variáveis analisadas pré e pós o jogo de basquetebol. *Diferença significativa dos valores pré em relação aos valores pós-partida $(p \leq 0,05)$. 


\section{DISCUSSÃO}

Os resultados encontrados mostram que os hormônios testosterona e cortisol aumentaram significativamente ao final da partida. Esses dois marcadores vêm sendo utilizados para verificar processos anabólicos e catabólicos durante o treinamento e/ou eventos esportivos específicos.

$V_{i r u^{17}}$ sugere que a resposta hormonal é diretamente relacionada ao trabalho total realizado pelo atleta e a correlação entre as concentrações de hormônio e performance é estabelecida posteriormente ao efeito hormonal no tecido muscular. Contudo, tem sido utilizada a relação entre variações hormonais e overtraining, principalmente quando é analisada a concentração de testosterona e cortisol ${ }^{18}$.

O aumento da concentração de testosterona plasmática se deve à atividade aumentada do eixo hipotálamo-hipófise-gonadal durante o exercício físico $^{18}$. Simões et $a^{7}$, em estudo com corredores velocistas e fundistas, analisaram a razão testosterona/ cortisol e sugeriram que a concentração de testosterona pode ajudar o atleta a se recuperar mais rapidamente entre as sessões de treinamento, permitindo assim a realização de um volume de treinamento específico. Neste sentido, é possível que no presente estudo, as concentrações aumentadas de testosterona após a partida de basquetebol estejam ligadas às adaptações orgânicas com objetivo de recuperação dos atletas. Por conseguinte, o controle da resposta deste hormônio pode indicar um efeito positivo da carga de treinamento aplicada, ao passo que, se a testosterona permanecer inibida por longos períodos, a recuperação dos atletas pode estar comprometida.

Outro hormônio que vem sendo estudado é o cortisol, porém, como marcador específico de catabolismo muscular, tendo sido observados aumentos significativos do mesmo quando a intensidade do exercício excede $60 \%$ do limiar anaeróbio ${ }^{6,17,19}$.

Segundo Simões et al ${ }^{7}$, em situações de estresse, quando o atleta é submetido a treinamento ou evento esportivo intenso, ocorre o aumento nas concentrações de dopamina cerebral e norepinefrina, desencadeando um aumento na secreção de fatores hormonais, estes promovem o aumento de hormônios hipofisários, resultando na maior secreção de cortisol pelo córtex da glândula adrenal. O basquetebol é caracterizado como uma atividade de alta intensidade, pela grande participação do metabolismo anaeróbio durante as partidas ${ }^{20}$.

Os aumentos dos níveis de cortisol podem aumentar a lipólise e o catabolismo protéico para mobilizar substratos energéticos durante e na recuperação pós-exercício ${ }^{21}$. O presente estudo corrobora o estudo conduzido por Hackney et $\mathrm{al}^{22}$, que apresentou aumento significante na resposta do hormônio cortisol após exercício anaeróbio. Sendo assim, o aumento no cortisol observado no presente estudo, pode estar relacionado às características intermitentes da modalidade basquetebol. No entanto, se concentrações de cortisol forem detectadas em concentrações muito elevadas por longos períodos (processo de treinamento anual) o desempenho atlético pode reduzir, bem como pode aumentar o aparecimento de lesões, indicando recuperação insuficiente e desgaste dos atletas.

Outra modificação observada foi nos parâmetros imunológicos, por meio do aumento significativo no número de leucócitos totais e neutrófilos. Esses parâmetros são importantes devido à influência que o exercício físico exerce ao sistema imunológico, podendo, em situações de sobrecarga excessiva, levar a mudanças negativas na imunidade do atleta e o aumento do risco de infecções do trato respiratório superior $^{10}$.

A resposta imune ao estresse do exercício envolve a coordenação de vários tipos de células, fatores de solubilidade e sinalizadores moleculares no sangue e nos tecidos ${ }^{23}$. As células imunológicas que respondem de maneira especializada são os linfócitos, capazes de reconhecer e distinguir diferentes antígenos de maneira mais específica. Os neutrófilos e macrófagos (monócitos) são responsáveis pela eliminação de organismos por fagocitose, sendo as primeiras células a responder a entrada de um antígeno ${ }^{24}$. Alterações nas funções dos neutrófilos e linfócitos parecem ser dependentes não somente da intensidade, mas também da duração do esforço ${ }^{25}$.

De forma geral, o exercício físico agudo provoca um aumento na concentração de leucócitos, decorrente principalmente do aumento da concentração de neutrófilos. Este aumento parece resultar da migração de células do endotélio para o sangue ou como parte da resposta inflamatória às lesões no tecido muscular. ${ }^{26,27}$. O aumento observado nos parâmetros imunológicos (leucócitos totais e neutrófilos) pode ter relação com o aumento nas concentrações de cortisol plasmático ${ }^{28}$. Visto que, o cortisol em pequenas quantidades estimula a migração de células da medula para a circulação que posteriormente são conduzidas para os tecidos lesionados ${ }^{29}$. Dentre as limitações do atual estudo, está o fato de as coletas terem sido realizadas apenas após a partida, não contando com outros períodos de análise (10, 24 e 48 horas após o evento). Em termos clínicos, é prematuro dizer que o aumento agudo nas células do sistema imune pode indicar uma melhora imunológica. Aspectos da funcionalidade celular devem ser analisados, como a citotoxidade de linfócitos e capacidade fagocitária de macrófagos e neutrófilos. Adicionalmente, a adaptação do sistema imune deve ser observada durante toda a temporada, com vistas a monitorar uma possível imunossupressão. No entanto, as dosagens agudas podem servir como método de monitorar a concentração de células, pois se houver uma queda brusca na contagem das mesmas, um menor número celular estará disponível para proteção imune do atleta.

Outro aspecto observado neste estudo foi o comportamento das enzimas LDH e CK, já que podem fornecer indicações do grau de adaptação metabólica do músculo esquelético, adaptações bioquímicas do organismo frente à carga de treinamento ${ }^{12}$ e o 
comportamento típico destas enzimas é o aumento após um evento desportivo ${ }^{30}$.

Os resultados apresentados neste estudo não foram estatisticamente significativos, entretanto os valores foram numericamente maiores quando comparados os momentos pré e pós-partida, mostrando que pode ter ocorrido o extravasamento das enzimas através das estruturas da membrana.

Contudo, tem sido reportado que o exercício eleva os níveis de enzimas a LDH e CK pelos possíveis mecanismos de inflamação muscular aumentando a permeabilidade da membrana celular ${ }^{30}$.

Esse aumento da atividade sérica de CK e $\mathrm{LDH}$ tem sido aceito como bons indicadores de dano muscular ${ }^{30}$. O fato de não terem sido observados aumentos estatisticamente significativos nestas enzimas pode estar ligado à questão dos atletas participarem sistemicamente de competições, sendo razoável hipotetizar que eles estavam adaptados a partidas intensas de basquetebol. Dentre os fatores que podem ter influenciado os resultados do presente estudo está o limitado número de atletas analisados.

\section{CONCLUSÃO}

A simulação de uma situação de jogo promove aumento significativo nas variáveis hormonais (testosterona e cortisol) e nas variáveis imunológicos (leucócitos totais e neutrófilos), indicando uma resposta orgânica adaptativa aguda dos atletas. Entretanto, apesar dessas mudanças nas variáveis hormonais e imunológicas, não foi observado mudança significativa nas enzimas em questão. Neste sentido, outros trabalhos a respeito desses marcadores na modalidade do basquetebol devem ser realizados. Como sugestão, indica-se a realização de dosagens em diferentes momentos após o estímulo, avaliação da funcionalidade das células imunológicas e avaliação das adaptações durante um processo de treinamento anual, além de um maior número de indivíduos a serem avaliados.

\section{REFERÊNCIAS BIBLIOGRÁFICAS}

1. Hoffman JR, Kang J, Ratamess NA, Faigenbaum $A D$. Biochemical and hormonal responses during an intercollegiate football season. Med Sci Sports Exerc 2005;37(7):1237-1241.

2. Smith DJ. A framework for understanding the training process leading to elite performance. Sports Med 2003;33(15):1103-1126.

3. Foster $\mathrm{C}$. Monitoring training in athletes with reference to overtraining syndrome. Med Sci Sports Exerc 1998;30(7):1164-1168.

4. Urhausen A, Kindermann W. The endocrine system in overtraining. In: Warren MP, Constantini NW. editors. Sports Endocrinology 2000. p. 347-370.

5. Eliakim A, Nemet D, Bar-Sela S, Higer Y, Falk B. Changes in Circulating IGF-I and their Correlation with Self-Assessment and Fitness Among Elite Athletes. Int J Sports Med 2002;23(8):600-603.

6. Banfi G, Marinelli M, Roi, GS, Agape,V. Usefulness of free testosterone/ cortisol ratio during season of elite speed skating athletes. Int J Sports Med 1993;14:373-379.

7. Simões HG, Marcon F, Oliveira F, Campbell CSG, Baldissera V.; Costa Rosa LFBP. Resposta da razão testosterona/cortisol durante o treinamento de corredores velocistas e fundistas. Rev Bras Educ Fis Esp 2004;18(1):31-46.

8. Izquierdo M, Ibañez J, Häkkinen K, Kraemer WJ, Ruesta $M$, Gorostiaga EM. Maximal strength and power, muscle mass, endurance and serum hormones in weightlifters and road cyclists. J Sports Sci 2004;22(5):465-478.

9. Mochida N, Umeda T, Yamamoto Y, Tanabe M, Kojima A, Sugawara K, et al. The main neutrophil and neutrophilrelated functions may compensate for each other following exercise - a finding from training in university judoists. Luminescence 2007 22:20-28.

10. Nieman DC. Current perspective on exercise immunology. Curr Sports Med Rep 2003;2(5):239-242.

11. Prestes J, Foschini D, Donatto FF. Efeitos do exercício físico sobre o sistema imune. Rev Bras Ciência Saúde 2006;1:57-65.

12. Brancaccio $P$, Limongelli FM, Maffulli N. Monitoring of serum enzymes in sport. Br J Sports Med 2006;40:96-97.

13. Foschini D, Prestes J, Charro MA. Relação entre exercício físico, dano muscular e dor muscular de início tardio. Rev Bras Cineantropom Desempenho Hum 2007;9(1):107-112.

14. Thompson PD, Crouse SF, Goodpaster B, Kelley D.; Moyna N, Pescatello L. The acute versus chronic response to exercise. Med Sci Sports Exerc 2001;33:S438-S445.

15. Rowbottom DG, Green KJ. Acute exercise effects on the imune system. Med Sci Sports Exerc 2000;32:S396-S405.

16. Afonso M, Souza CN, Zanatto AM, Luciano E. Respostas metabólicas agudas ao exercício físico moderado em ratos wistar. Motriz 2003;9(2):87-92.

17. Viru A. Hormonal functions and exercise. In Current: Therapy in Sports Medicine, St. Louis, MO: MosbyYearBook, 3rd, 1995 p.501-506.

18. Gabriel HHW, Urhausen A, Kindermann W. Impaired pituitary hormonal response to exhaustive exercise in overtrained endurance athletes. Med Sci Sports Exerc 1998;30(7):407-14.

19. Jürimäe $\mathrm{J}$, Jürimäe $\mathrm{T}$, Purge $P$. Plasma testosterone and cortisol responses to prolonged sculling in male competitive rowers. J Sports Sci 2001;19(11):893898.

20. Kokubun E, Daniel JF. Relações entre a intensidade e duração das atividades em partida de basquetebol com as capacidades aeróbica e anaeróbica: estudo pelo lactato sangüíneo. Rev Paul Educ Fís 1992;6:37-46.

21. Nindl BC, Kraemer WJ, Deaver DR, Peters JL, Marx JO, Heckman JT, et al. LH secretion and testosterone concentrations are blunted after resistance exercise in men. J Appl Physiol 2001;91:1251-1258.

22. Hackney AC, Premo MC, Mcmurray RG. Influence of aerobic versus anaerobic exercise on the relationship between reproductive hormones in men. J Sports Sci 1995;113(4):305-311.

23. Venkatraman JT, Pendergast DR. Effect of Dietary Intake on Immune Function in Athletes. Sports Med 2002;32(5):323-337.

24. Castell LM. Can glutamine modify the apparent immunodepression observed after prolonged, exhaustive exercise? Nutrition 2002;18(5):371-375.

25. Leandro C, Nascimento E, Manhães-de-castro R, 
Duarte JÁ, De-castro CMMB. Exercício físico e sistema imunológico: mecanismos e integrações. Rev Port Cien Desp 2002;2:80-90.

26. Mccarthy DA, Dale MM. The leucocytosis of exercise. A review and model. Sports Med. 1988;6:333-363.

27. Smith LL. Acute inflammation: the underlying mechanism in delayed onset muscle soreness? Med Sci Sports Exerc. 1991;23(5):542-551.

28. Ottaway CA, Husband AJ. The influence of neuroendocrine pathways on lymphocyte migration.
Immunol Today 1994;15(11):511-517

29. Brenner I, Shek PN, Zamecnik J, Shephard RJ. Stress Hormones and the immunological responses to heat and exercise. Int J Sports Med 1998;10:130-143.

30. Schroder H, Navarro E, Mora J, Galiano D, Tramullas A. Effects of $\alpha$-tocopherol, B-carotene and ascorbic acid on oxidative, hormonal and enzymatic exercise stress markers in habitual training activity of professional basketball players. Eur J Nutrition 2001; 40:178-184.

\section{Endereço para correspondência}

Jonato Prestes

Rua Major José Inácio, $n^{\circ}$. 2400. Edifício Ouro Preto

Recebido em 10/10/07

- Apartamento 13.

Revisado em 09/01/08

CEP 13560-161 - São Carlos-SP.

Aprovado em 03/02/08

E-mail: jonatop@gmail.com 